\title{
Autofahren mit Diabetes birgt meist kein relevant erhöhtes Unfallrisiko
}

\author{
Ein Diabetes mellitus kann sich auf die Eignung zum Führen von Kraftfahrzeugen auswirken. \\ Doch meistens können Menschen mit Diabetes trotzdem sicher PKW und LKW fahren. In Einzel- \\ fällen - vor allem bei rezidivierenden Hypoglykämien - muss der Arzt aber zumindest vorüber- \\ gehend das Autofahren untersagen.
}

Jeder zehnte Autofahrer in Deutschland ist Diabetiker. „Im Einzelfall können unvorhergesehene Unterzuckerungen ebenso wie Hyperglykämien die Fahrtauglichkeit beeinflussen“, erläuterte der Vorsitzende des Ausschusses Soziales der Deutschen Diabetes Gesellschaft Oliver Ebert, Rechtsanwalt in Stuttgart. Nach aktueller Datenlage bestehe für sie jedoch insgesamt kein relevant erhöhtes Risiko für einen Verkehrsunfall. Im Gegenteil, die erhöhte Vorsicht und Sorgfalt bei diesen Patienten könne sogar dazu führen, dass das Unfallrisiko geringer sei.

\section{Hyper- und Hypoglykämien sind gefährlich}

Doch bei schlecht eingestellten Patienten mit dauerhaft extrem erhöhten Blutzuckerwerten kann die Fahreignung schon beeinträchtigt sein; denn solche Hyperglykämien sind mit Konzentrationsstörungen und/oder temporären Beeinträchtigungen des Sehvermögens assoziiert.

Besonders gefährlich sind aber die plötzlich auftretenden Hypoglykämien bei medikamentös behandelten Diabetikern. Betroffen sind vorrangig Patienten, die mit Sulfonylharnstoffen oder Insulin behandelt werden. Metformin, SGLT2-Hemmer und inkretinbasierte Therapien mit Gliptinen oder GLP-1-Analoga verursachen in der Monotherapie dagegen normalerweise keine Unterzuckerungen. Die entscheidenden Unfallursachen in diesem Zusammenhang sind meist Fehleinschätzungen oder Fehler beim Selbstbehandlungsverhalten wie eine falsche Insulin- oder Medikamentendosierung, Verwechslung des Insulinpräparates, Alkoholkonsum oder unzureichende Blutzuckerselbstkontrollen. „Insgesamt besteht allerdings kein relevant erhöhtes Risiko“ so Ebert.

Dies belegen Daten einer deutschen Studie. Danach liegt die Unfallrate aufgrund von Hypoglykämien nur bei 0,001 bis 0,49 pro 100.000 Kilometer oder 0,007 bis 0,01 pro Jahr. Daraus ergibt sich, dass im Mittel ein Unfall in Folge einer Unterzuckerung erst nach einer Fahrleistung von ca. 400.000 Kilometern beobachtet werden konnte [1]. Ähnlich sind die Ergebnisse anderer internationaler Studien. Und dies gilt auch für Patienten, die mit Insulin behandelt werden. „Auch im Vergleich zu Krankheiten wie ADHS oder obstruktiver Schlafapnoe ist das relative Unfallrisiko bei Diabetikern erheblich geringer“, so Ebert. Daten von Krankenversicherungen zeigten sogar eine Tendenz zur Reduktion von Unfällen am Arbeitsplatz bei Diabetikern. Dies spreche dafür, dass Betroffene in Kenntnis des Gefahrenpotenzials sogar eine gesteigerte Aufmerksamkeit und Sorgfalt walten lassen. Und dies gelte sowohl für den Arbeitsplatz als auch im Straßenverkehr.

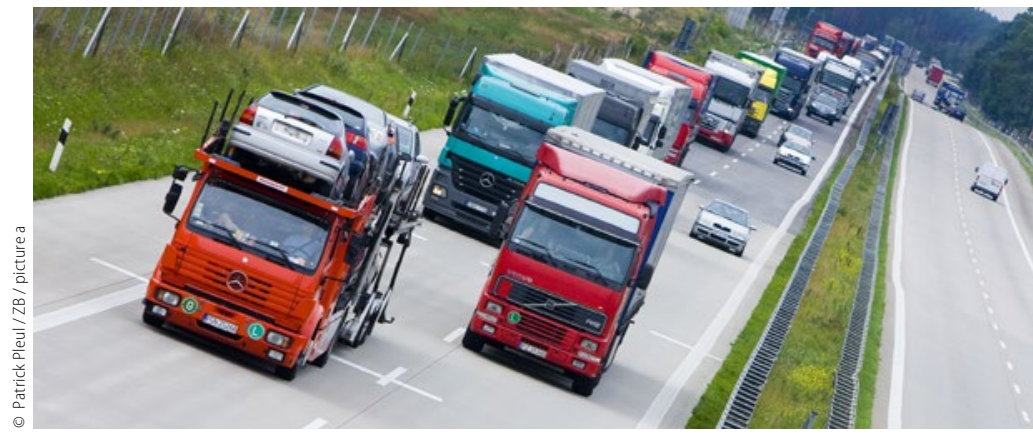

Gut eingestellt und geschult steht mit Diabetes auch dem Führen eines LKWs nichts entgegen, wenn das Hypoglykämieempfinden ok ist.

\section{Keine Einschränkungen bei guter Einstellung}

Nach den von der Bundesanstalt für Straßenwesen herausgegebenen Begutachtungsleitlinien können deshalb gut eingestellte und ausreichend geschulte Diabetiker sowohl PKW als auch LKW sicher führen. Dies gilt auch für die Personenbeförderung mit Taxi oder Omnibus. Voraussetzung allerdings ist, dass die Hypoglykämiewahrnehmung nicht beeinträchtigt ist. Kommt es innerhalb von zwölf Monaten wiederholt zu fremdhilfebedürftigen Unterzuckerungen, so sollte der Patient bis auf Weiteres nicht mehr am Straßenverkehr teilnehmen. „In solchen Fällen muss der behandelnde Arzt ausdrücklich vom Autofahren abraten", so Ebert.

Eine Teilnahme am Straßenverkehr sei allerdings dann wieder möglich, sobald eine hinreichende Stabilität der Stoffwechseleinstellung und eine zuverlässige Wahrnehmung von Hypoglykämien sichergestellt seien. Dies erfordere eine intensive Schulung und auch ein Hypoglykämie-Wahrnehmungstraining. Auch eine Umstellung der Therapie, eine Intensivierung der Selbstmessungen oder auch der Einsatz eines kontinuierlichen Glukosemonitoringsystems kann hilfreich sein. Auch muss garantiert sein, dass es künftig nicht mehr zu dem Fehlverhalten kommt, welches die Hypoglykämie bzw. den Autounfall ausgelöst hat. Dann sei auch nach einem Unfall die Teilnahme am Straßenverkehr wieder möglich. Dr. med. Peter Stiefelhagen

\footnotetext{
Literatur

1. Harsch I A et al. J Intern Med 2002: 252(4): 352-60
}

Quelle: 10. Herbsttagung der Deutschen Diabetesgesellschaft (DDG), 4. November 2016 in Nürnberg 